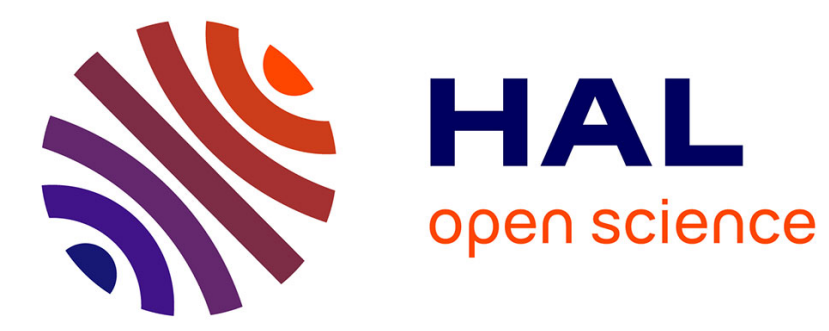

\title{
Turbulence statistics downstream of a vorticity generator at low Reynolds numbers
}

Charbel Habchi, Thierry Lemenand, Dominique Della Valle, Hassan

Peerhossaini

\section{- To cite this version:}

Charbel Habchi, Thierry Lemenand, Dominique Della Valle, Hassan Peerhossaini. Turbulence statistics downstream of a vorticity generator at low Reynolds numbers. Physics of Fluids, 2016, 28 (10), Non spécifié. 10.1063/1.4964924. hal-02525552

HAL Id: hal-02525552

https://univ-angers.hal.science/hal-02525552

Submitted on 31 Mar 2020

HAL is a multi-disciplinary open access archive for the deposit and dissemination of scientific research documents, whether they are published or not. The documents may come from teaching and research institutions in France or abroad, or from public or private research centers.
L'archive ouverte pluridisciplinaire HAL, est destinée au dépôt et à la diffusion de documents scientifiques de niveau recherche, publiés ou non, émanant des établissements d'enseignement et de recherche français ou étrangers, des laboratoires publics ou privés. 


\title{
Turbulence statistics downstream of a vorticity generator at low Reynolds numbers
}

\author{
Charbel Habchi, ${ }^{1}$ Thierry Lemenand, ${ }^{2}$ Dominique Della Valle, ${ }^{3}$ \\ and Hassan Peerhossaini ${ }^{4}$ \\ ${ }^{1}$ Mechanical Engineering Department, Notre Dame University - Louaize, \\ Zouk Mosbeh, Lebanon \\ ${ }^{2}$ Université d'Angers, ISTIA, LARIS EA 7315, Angers, France \\ ${ }^{3}$ Food Processing Department, ONIRIS, GEPEA, Nantes, France \\ ${ }^{4}$ Astroparticles and Cosmology Laboratory (CNRS-UMR 7164), Energy Physics Group, \\ Université Sorbonne Paris Cité, Paris, France
}

(Received 26 October 2015; accepted 4 October 2016; published online 24 October 2016)

\begin{abstract}
Vortex generators (VGs) are inserted in turbulent pipe flows in order to improve mixing and heat and mass transfer while a moderate pressure drop is maintained. The purpose of the present study is to contribute to the elaboration of scaling laws for the turbulence decay downstream a row of VGs. This knowledge will help in the design of such systems, especially for optimal geometry and spacing of the VG. The experimental study is carried out using laser Doppler anemometry at different locations downstream of the row of VGs so as to probe the streamwise velocity field. The Taylor microscale Reynolds number $\mathrm{Re}_{\lambda}$ ranges between 15 and 80 so that, for the lowest flow rates, fully developed turbulence conditions are not fulfilled. Comparison of the integral length scale to data in the open literature shows that the conventional scaling laws at the dissipative scale are fairly assessed. It is shown that the turbulence macroscale increases in the streamwise direction and is scaled by the VG dimensions. The normalized turbulent energy dissipation rate has values between 0.5 and 2.8 , with -1 power-law decay as a function of the Taylor microscale Reynolds number. This observation is consistent with previous findings using direct numerical simulations (DNS). The streamwise variation of the turbulence energy dissipation rate shows an exponential decay; it reaches an asymptotic value after a distance of about 6 times the VG height. Published by AIP Publishing. [http://dx.doi.org/10.1063/1.4964924]
\end{abstract}

\section{INTRODUCTION}

Vorticity is an efficient means of mixing intensification for multifunctional heat exchangers/ reactors, so that vortex generators (VGs) are frequently used in order to enhance the heat, mass, and momentum transfers in laminar and turbulent flows. The dynamics and production mechanisms of these coherent structures and their effects on the transport phenomena are the subject of numerous studies in the literature. ${ }^{1-5}$ In fact, the turbulent large scale motion is not universal and depends strongly on the local flow structure, ${ }^{6,7}$ unlike the small-scale eddies that exhibit universal characteristics by auto-similarity. ${ }^{8}$ However, to the best of our knowledge, turbulence decay and scaling laws in the flow behind VGs have rarely been studied (compared to, for instance, free jets, isotropic and anisotropic turbulence, elastic turbulence, etc. ${ }^{9-14}$ ). Such an analysis is fundamental to study the meso- and micromixing processes in multifunctional heat exchangers/reactors, which are related to the integral length scale and to the turbulent energy dissipation rate. ${ }^{15,16}$ For instance, investigations on grid-generated turbulence suggest that a very rapid decay of the turbulent energy dissipation takes place in the very thin layer located immediately after the grid, and that drop breakup (in two-phase flows) and micromixing are therefore expected to dominate in this high-energy dissipation region. On the other hand, coalescence becomes significant further downstream where low turbulent energy dissipation rates prevail. ${ }^{17,18}$ 


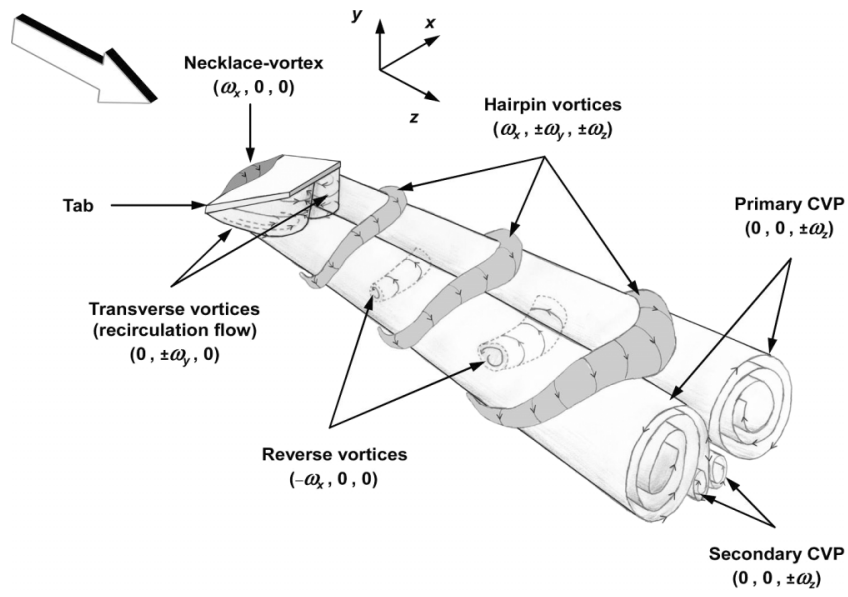

FIG. 1. Schematic of the main flow structures generated by a trapezoidal VG. $\left(\omega_{x}, \omega_{y}, \omega_{z}\right)$ are the vorticity components in the Cartesian coordinate system. Reproduced with permission from Habchi et al., J. Turbul. 11, N36 (2010). Copyright 2010 Taylor \& Francis Ltd.

The present study focuses on the distribution of the integral length scale and the turbulent energy dissipation rate in the wake of the VG row. The global Reynolds numbers considered here range from 7500 to 15000 , corresponding to common operating conditions of VGs. ${ }^{19,20}$ The results obtained in the present study are compared to other experiments and direct numerical simulations (DNS) data from the open literature.

The flow configuration consists of a straight circular pipe in which four VGs are inserted in a quadrant arrangement in the tube cross section. Each VG consists of a trapezoidal plate inclined to the wall with a $30^{\circ}$ angle in the flow direction. Each VG generates a complex vortical flow (see Figure 1), which becomes the single leading mechanism for the heat and mass transfer. ${ }^{21,22}$ A steady counter-rotating vortex pair (CVP) is formed in the wake of the VG due to the pressure difference between the low-momentum region under the VG and the high-momentum region above the VG, in the flow core. Moreover, a three-dimensional shear layer develops around the vortex generator starting from its leading edge. This shear layer becomes unstable downstream and generates hairpin-like structures, due to the Kelvin-Helmholtz instability in a free shear flow. Primary hairpins can further generate secondary instabilities in the form of reverse vortices in the tab wake. ${ }^{2}$

The paper is organized as follows: Section II describes the experimental setup using the LDA system and the data acquisition. The experimental results on mixing length and energy dissipation rate are presented in Section III, as well as their modeling by scaling laws. In Sec. IV, some remarks and prospects are given concerning vorticity promoters in turbulent flows.

\section{EXPERIMENTAL SETUP AND DATA ANALYSIS METHODS}

The test section is a straight circular pipe of $20 \mathrm{~mm}$ inner diameter in which one row of four mixing tabs is fixed in a quadrant. The tabs are inclined $30^{\circ}$ with respect to the tube wall, as shown in Figure 2. The test section is preceded by a preconditioner (200 $\mathrm{cm}$ straight Plexiglas pipe) in order to generate a fully developed flow at the test section inlet, and is followed by a postconditioner (20 cm straight Plexiglas pipe). The connections between the different elements are carefully designed to avoid any protuberance that could disturb the flow. A pulsation damper is added to the flow loop to limit the pressure fluctuations produced by the pump.

Measurements are made using a Dantec Dynamics@ laser Doppler anemometry (LDA) system equipped with a $10 \mathrm{~W}$ argon-ion laser source and two BSA-enhanced signal-processing units (57N20 BSA and 57N35 BSA enhanced models); the measuring head is equipped with a $160 \mathrm{~mm}$ focal lens. The laser wavelength is $514.5 \mathrm{~nm}$ (green) and the shift frequency is $40 \mathrm{MHz}$ (Bragg cell). The laser beam separation distance is $40 \mathrm{~mm}$ and its diameter before the lens is $3.8 \mathrm{~mm}$; 


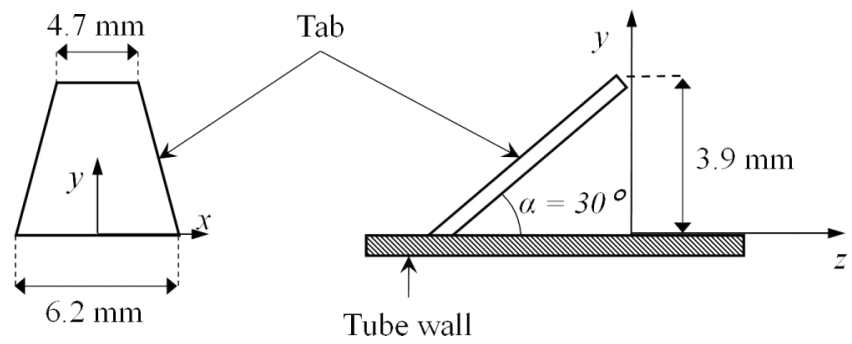

FIG. 2. Dimensions of the VG and the Cartesian coordinate system.

the beam intersection angle is $13.55^{\circ}$. The measurement volume of the LDA is positioned by a three-dimensional lightweight precision traversing system controlled via a PC. Precision of the measurement volume positioning system is $12.5 \mu \mathrm{m}$. Measurement volume dimensions are $404 \mu \mathrm{m}$ length, $48 \mu \mathrm{m}$ width and height, and it contains 21 fringes spaced by $2.18 \mu \mathrm{m}$. Due to the cylindrical shape of the pipe, in order to avoid light-beam refraction only the axial component of the Reynolds stress and mean velocity are measured in the radial direction. Flow is seeded by small mica particles coated in titanium oxide of size between 0.1 and $0.5 \mu \mathrm{m}$. Their density is $3 \mathrm{~g} \mathrm{~m}^{-3}$ and they have a response frequency of $1 \mathrm{kHz}$.

The measurement time ranges between $60 \mathrm{~s}$ and $360 \mathrm{~s}$, which is about $6 \times 10^{3}$ to $36 \times 10^{3}$ times the integral time scale, thus ensuring statistical convergence. During this measurement time, around 30000 validated sampling particles were obtained. The data-acquisition rate is in the range $1-4 \mathrm{kHz}$.

Calibration of the LDA system, including Bragg-cell oscillation and orientation sensitivity, light beam power, reference point, and optics alignment, was performed. The precision error was estimated at about $2.5 \%$. Moreover, to ensure the reproducibility of the LDA measurements, experiments were iterated four times for radial profiles at different positions for Reynolds number 15000 . It was found that the reproducibility error did not exceed 6\%.

Using the sampling rate uncertainty method of Benedict and Gould, ${ }^{23}$ the confidence level is determined to be $95 \%$; in addition, $15 \%$ of the measured data had an error less than $1 \%$ and $85 \%$ less than $10 \%$.

The integral time scale is evaluated from the temporal correlation function, with a single probe for the velocity measurements. Hence, the sampling must be performed at sufficiently short time intervals to detect high-frequency fluctuations. LDA allows such fast measurements by optimizing seeding and optical adjustments. However, since the LDA measurements are not carried out at constant time intervals, the data must be resampled according to the Høst-Madsen and Caspersen ${ }^{24}$ method.

\section{RESULTS AND DISCUSSION}

\section{A. Operating conditions and global results}

Measurements are performed in the wake of the VG, at $y / h=0.5$, for different axial locations $z / h=0.75 ; 1.5 ; 2.5 ; 6.25$, and 8.75 , as shown schematically in Figure 3, for the Reynolds numbers [7500-10 000-12 500-15 000]. Turbulence properties at different Reynolds numbers and measurement stations are summarized in Table I.

\section{B. Turbulence power spectra}

The dimensionless energy spectra $E /\left(\eta u_{\eta}^{2}\right)$, with $\eta$ and $u_{\eta}$ respectively the Kolmogorov scale and the Kolmogorov velocity, for the different axial locations $z / h$ in the VG wake, are presented in Figure 4 and are compared with Pao's ${ }^{25}$ energy spectrum. Taylor microscale Reynolds numbers vary in the range $15<\operatorname{Re}_{\lambda}<80$. 


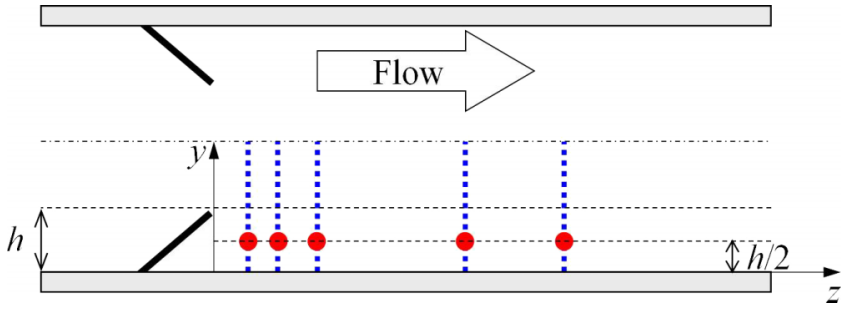

FIG. 3. Measurement points (red dots) on the radial profiles in the tab symmetry plane.

The Kolmogorov microscale $\eta=\left(v^{3} / \varepsilon\right)^{1 / 4}$ is computed from the turbulence energy dissipation rate given by ${ }^{26}$

$$
\varepsilon=15 v \overline{(\partial u / \partial z)^{2}} .
$$

Following the Taylor hypothesis, ${ }^{10,27}$ one can write $\partial u / \partial z=\left(1 / U_{c}\right) \times(\partial u / \partial t)$ with $U_{c}$ the convective velocity defined for three-dimensional flows by $U_{c}^{2}=U^{2}\left(1+5 \overline{u^{\prime 2}} / U^{2}\right)$, with $U$ the mean velocity and $\overline{u^{\prime 2}}$ the first diagonal component of the Reynolds stress tensor.

The inset in Figure 4 shows the compensated velocity spectra $E /\left(\eta^{-2 / 3} u_{\eta}^{2} k^{-5 / 3}\right)$ as functions of the dimensionless wave number. In the large scales (small wave numbers), and according to Kolmogorov's similarity hypothesis, the compensated spectra tend to a constant value ${ }^{8}$

$$
C_{K}=\frac{E}{k^{-5 / 3} \eta^{-2 / 3} u_{\eta}^{2}}
$$

where $C_{K}$ is the Kolmogorov universal constant. In the inset, it is observed that the actual results for the VG wake lie in the Kolmogorov constant range between 0.33 and 0.69 according to the analysis of Sreenivasan, ${ }^{28}$ giving a Kolmogorov constant of 0.53 with standard deviation 0.055 when $\operatorname{Re}_{\lambda}$ exceeds 50 . The present data, obtained for $15<\operatorname{Re}_{\lambda}<80$, are consistent as they show a mean value

TABLE I. Turbulence properties at various Reynolds numbers and measurement stations. Here, $f_{\mathrm{K}}$ is the Kolmogorov frequency.

\begin{tabular}{lccccccccc}
\hline \hline$U(\mathrm{~m} / \mathrm{s})$ & $\frac{z}{h}$ & $\mathrm{Re}_{z}$ & $\mathrm{Re}_{\lambda}$ & $\frac{u}{U}$ & $\frac{L}{h}$ & $\varepsilon\left(\mathrm{m}^{2} / \mathrm{s}^{3}\right)$ & $\frac{\eta}{h} \times 10^{3}$ & $f_{\mathrm{K}}=\frac{U}{2 \pi \eta}(\mathrm{Hz})$ & $\frac{u_{\eta}}{U}$ \\
\hline \multirow{5}{*}{0.375} & 0.77 & 1125 & 16 & 1.12 & 0.14 & 3.38 & 6 & 535 & 0.55 \\
& 1.54 & 2250 & 20 & 0.59 & 0.15 & 2.19 & 6.7 & 914 & 0.26 \\
& 2.56 & 3750 & 42 & 0.58 & 0.25 & 2.04 & 6.8 & 1287 & 0.18 \\
& 6.41 & 9375 & 26 & 0.17 & 0.34 & 0.21 & 12 & 1086 & 0.07 \\
& 8.97 & 13125 & 34 & 0.15 & 0.93 & 0.08 & 15.2 & 926 & 0.05 \\
& 0.77 & 1500 & 26 & 1.31 & 0.15 & 3.89 & 5.8 & 617 & 0.51 \\
0.5 & 1.54 & 3000 & 30 & 0.6 & 0.2 & 2.76 & 6.3 & 1213 & 0.22 \\
& 2.56 & 5000 & 33 & 0.32 & 0.38 & 2.1 & 7.2 & 1432 & 0.12 \\
& 6.41 & 12500 & 38 & 0.18 & 0.93 & 0.33 & 10.7 & 1569 & 0.06 \\
& 8.97 & 17500 & 43 & 0.15 & 1.56 & 0.15 & 13.1 & 1398 & 0.04 \\
& 0.77 & 1875 & 50 & 1.53 & 0.26 & 2.68 & 6.3 & 613 & 0.42 \\
0.625 & 1.54 & 3750 & 56 & 0.62 & 0.4 & 1.91 & 6.9 & 1342 & 0.16 \\
& 2.56 & 6250 & 49 & 0.36 & 0.44 & 1.4 & 7.4 & 1872 & 0.1 \\
& 6.41 & 15625 & 61 & 0.17 & 0.84 & 0.27 & 11.3 & 1928 & 0.04 \\
& 8.97 & 21875 & 70 & 0.14 & 0.91 & 0.14 & 13.3 & 1773 & 0.03 \\
& 0.77 & 2250 & 61 & 0.85 & 0.21 & 5.5 & 5.3 & 1737 & 0.21 \\
& 1.54 & 4500 & 76 & 0.49 & 0.26 & 2.92 & 6.2 & 2466 & 0.11 \\
& 2.56 & 7500 & 77 & 0.32 & 0.35 & 1.45 & 7.4 & 2653 & 0.07 \\
& 6.41 & 18750 & 75 & 0.18 & 0.73 & 0.42 & 10 & 2562 & 0.04 \\
& 8.97 & 26250 & 78 & 0.15 & 0.7 & 0.23 & 11.7 & 2324 & 0.03 \\
\hline \hline
\end{tabular}




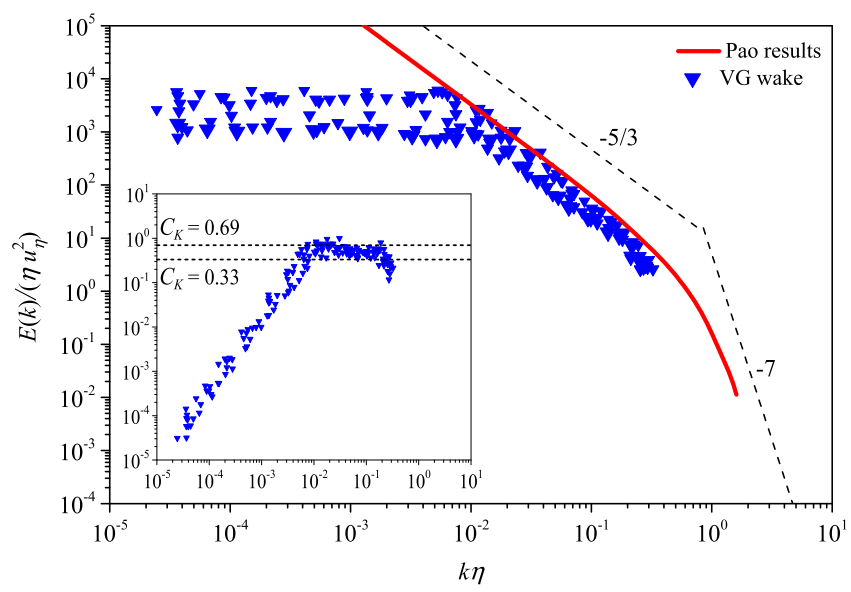

FIG. 4. One-component normalized energy spectra in the VG wake compared to the $\mathrm{Pao}^{25}$ spectrum. The inset shows the compensated power spectra plotted for the same flow in the VG wake.

of the Kolmogorov constant equal to 0.52 with standard deviation 0.10 . As expected, the spectra for large scales lack a universal behavior and instead depend on the flow configuration.

In the turbulent cascade, all the spectra are superimposed, as predicted by Kolmogorov's first similarity. Also, the spectra follow a $-5 / 3$ power-law until the Kolmogorov length. For higher wave numbers, the -7 power index characterizes the dissipation range dominated by viscous forces. ${ }^{7}$

\section{Integral length scale analysis}

The integral length scale $L$ of the most energetic turbulent eddies is fundamental for understanding the flow features as the development of boundary layers shed from bluff bodies, ${ }^{29}$ heat transfer ${ }^{30}$ or mesomixing processes. ${ }^{31}$

The integral scale $L$ is defined by the normalized temporal autocorrelation function $C(t)$ for the axial fluctuating velocity

$$
L=\int_{0}^{\infty} C(t) d t .
$$

The integral scale was computed here by a straightforward method using the velocity signal, sampled at a convenient frequency between 1 and $4 \mathrm{kHz}$.

The longitudinal Taylor microscale $\lambda$ is obtained theoretically from the second derivative of $C(t)$ at $t=0$. After some mathematical development, ${ }^{22} \lambda$ can be expressed as

$$
\lambda=\sqrt{\frac{\overline{u^{\prime 2}}}{\left(\partial u^{\prime} / \partial z\right)^{2}}},
$$

where $\partial u^{\prime} / \partial z=\left(1 / U_{c}\right)\left(\partial u^{\prime} / \partial t\right)$ by using the Taylor hypothesis as for Equation (1), so that the spatial derivative can be estimated by the temporal data.

The Taylor microscale Reynolds number $R e_{\lambda}$ is defined by

$$
\operatorname{Re}_{\lambda}=\frac{u^{\prime} \lambda}{v}
$$

In the present paper, the ratio $L / \eta$ is fitted by the power law

$$
\frac{L}{\eta}=0.206 \operatorname{Re}_{\lambda}^{1.37}
$$




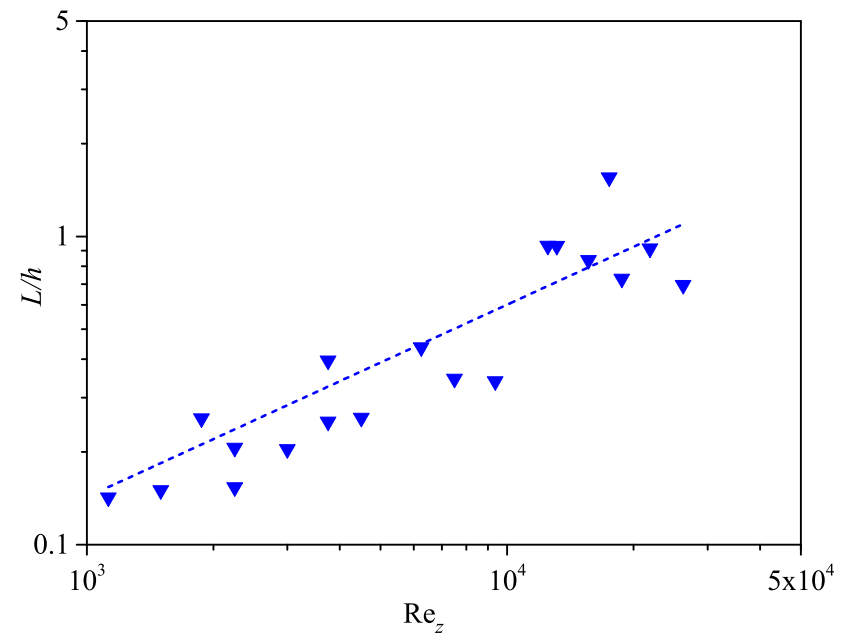

FIG. 5. Longitudinal integral scale normalized by the VG height versus dimensionless abscissa $\operatorname{Re}_{z}$.

This expression is to be compared with the models in the literature collected by Tsuji ${ }^{6}$ for experiments and DNS, respectively, $L / \eta=0.257 \operatorname{Re}_{\lambda}^{1.37}$ and $L / \eta=0.128 \operatorname{Re}_{\lambda}^{1.37}$. The discrepancies in the pre-factors reflect the dependence of the large eddy motion on the global flow geometry.

As reported by Tsuji, ${ }^{6}$ the integral length scale is not convenient for representing the beginning of the turbulent cascade (i.e., the higher scales of the inertial subrange), as the energy redistribution may not be achieved in case of strong anisotropy at the macroscale.

In fact, in the range of low Reynolds numbers studied here, the variation of turbulence properties with the mean flow velocity is not significant. However, it is much more significant in the streamwise direction. Therefore, a dimensionless abscissa is defined as follows:

$$
\operatorname{Re}_{z}=\frac{U z}{v}
$$

The integral scale normalized by a VG typical size (here we take the height, $h$ ) is plotted in Figure 5 as a function of the dimensionless abscissa $\operatorname{Re}_{z}$. In Figure 5 it is observed first that

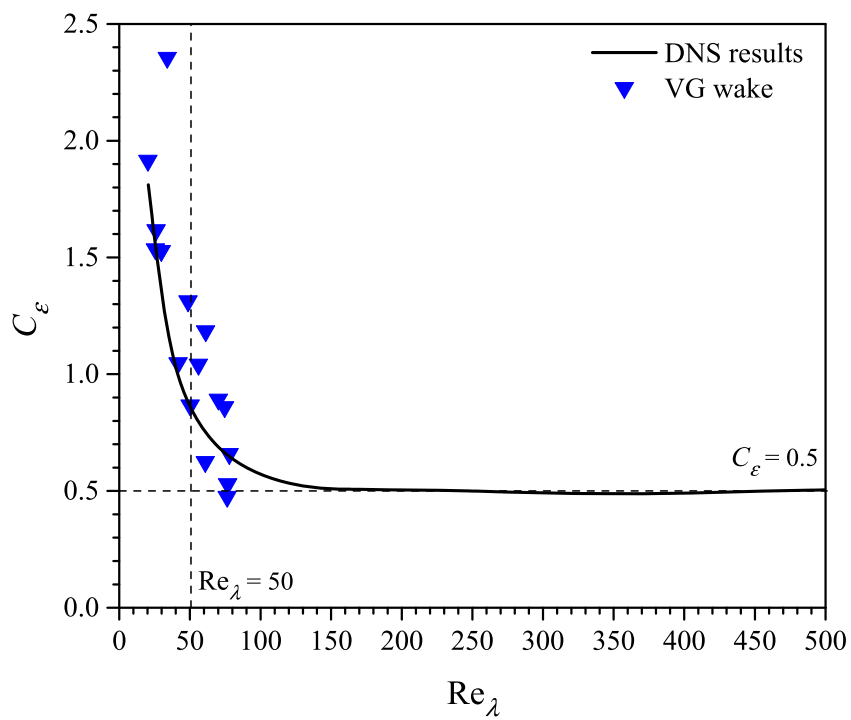

FIG. 6. Normalized turbulent energy dissipation rate $C_{\varepsilon}$ variation versus Taylor microscale Reynolds number $\operatorname{Re}_{\lambda}$ for the measured locations in the VG wake compared to DNS ${ }^{35-38}$ results. 


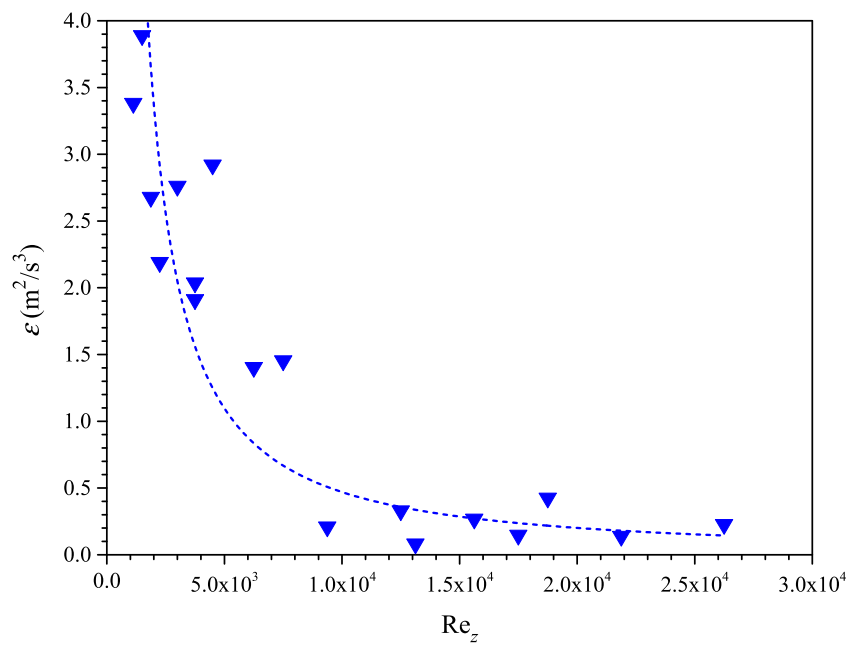

FIG. 7. Turbulent energy dissipation rate $\varepsilon$ variation versus Reynolds number $\operatorname{Re}_{z}$ based on the mean flow velocity and the $z$ coordinate.

the dimensionless integral scales are of the order of 0.5 (the values are spread between 0.1 and 1) demonstrating that the VG dominates the flow structure. Secondly, the integral scale expands downstream from the VG which is consistent with the observation of the flow topology (Figure 1). The integral scale probably reaches a plateau further downstream at a given level fixed by the equilibrium turbulence in the plain tube. Equation (8) represents the power-law fitting curve of the dimensionless scale $L / h$,

$$
\frac{L}{h}=0.002 \operatorname{Re}_{z}^{0.624}
$$

\section{Turbulence decay downstream of the VG}

Extensive studies are conducted to understand the dependence of the normalized turbulent energy dissipation rate $C_{\varepsilon}$, defined in Equation (9), on the Taylor microscale Reynolds number $\operatorname{Re}_{\lambda} \cdot{ }^{18,26,32,33}$ It is theoretically established that for $\operatorname{Re}_{\lambda} \geq 50, C_{\varepsilon}$ becomes constant with a value around $0.5 .^{26,32,33}$ However, for smaller $\operatorname{Re}_{\lambda}, C_{\varepsilon}$ exhibits a certain scattering, ranging between 0.5 and $2.5,{ }^{34}$

$$
C_{\varepsilon}=\frac{\varepsilon L}{{\overline{u^{\prime 2}}}^{3 / 2}} .
$$

In this section, $\mathrm{DNS}^{35-38}$ results are compared to the data obtained in the VG wake for $15<\operatorname{Re}_{\lambda}<80$ as shown in Figure 6. It can be observed from this figure that the values of $C_{\varepsilon}$ in a VG wake follow a power-law decay $C_{\varepsilon}=24 \operatorname{Re}_{\lambda}^{-0.8}$ (while Sreenivasan ${ }^{33}$ predicted a decay of the form $C_{\varepsilon}=18.8 \mathrm{Re}_{\lambda}^{-1}$ ) and approaches the value of 0.5 .

While the DNS results show a clear convergence to $C_{\varepsilon}=0.5$ for $\operatorname{Re}_{\lambda} \geq 50$, the experimental results show a wide discrepancy in $C_{\varepsilon}$, even for the same flow field, that is caused by some differences in the boundary conditions, as pointed out by Burattini et al. ${ }^{34}$

The turbulence decay downstream from the VG is investigated by looking at the variation of the turbulent energy dissipation rate $\varepsilon$ versus the streamwise dimensionless abscissa $\operatorname{Re}_{z}$ in Figure 7 . The energy dissipation rate decays (Eq. (10)) as one moves far from the VG (where the length scales increase as observed in Figure 5),

$$
\varepsilon=\varepsilon_{0} \operatorname{Re}_{z}^{\beta}
$$

with $\varepsilon_{0}=37.0 \times 10^{3}$ and $\beta=-1.23$. 
This decay is caused by the viscous effects acting on the large energy-containing eddies. Similar behavior was observed downstream of screen-type static mixers. ${ }^{39}$ In multifunctional heat exchangers/reactors, the turbulence energy dissipation rate is a key issue for the micromixing process since it is inversely proportional to the micromixing time, which in turn is related to the chemical reaction selectivity. ${ }^{15}$ Thus, to attain better chemical reaction selectivity, the dissipation rate $\varepsilon$ should be maintained relatively high depending on the chemical reaction time. ${ }^{16}$ For instance, it is observed that $\varepsilon$ drops to low values, below $0.5 \mathrm{~m}^{2} \mathrm{~s}^{-3}$, when $\mathrm{Re}_{z}$ exceeds 9.000 , corresponding to a distance of about $6 h$ downstream from the VG. Thus, adding a second array of VG at a distance $6 h$ from the previous one is recommended in order to maintain acceptable values of $\varepsilon$ and thus a good micromixing process.

\section{CONCLUSIONS}

The integral length scale is an important characteristic of the mesomixing process, which is the limiting procedure for micromixing. In fact, mesomixing is the process of disintegration of large eddies in the inertial sub-range of the energy cascade. Rapid mesomixing is important for fast chemical reactions. For instance, in multifunctional heat exchangers/reactors, when the scale of the injected stream of the secondary reagent is larger than that of the turbulent eddies, the concentration fluctuations break down from the integral scale to the Kolmogorov's microscale. Thus, the mesomixing time increases with the integral length scale. Therefore, it is desirable to keep the integral length scales as small as possible for fast chemical reactions. Thus, the size of the injected stream of the secondary reagent and its location can be fixed according to the integral length scale. In the present study, the evolution of the integral length scale versus $\operatorname{Re}_{\lambda}$ shows good agreement with the data from the literature. The development of the integral scale behind the vortex generator provides a consistent representation, considering that the flow structure is governed by the large-scale motion.

Knowledge of the local dissipation rate is also important in the design of multifunctional systems, since it governs the micromixing process. High levels of turbulence should be maintained for fast chemical reactions. The present work analyzes the evolution of the normalized turbulent energy dissipation rate $C_{\varepsilon}$ downstream of the VG array. It is shown that in the range $15<\operatorname{Re}_{\lambda}<80$, $C_{\varepsilon}$ has scatter between 0.5 and 2.8. It is also shown that when $\operatorname{Re}_{\lambda}$ increases, $C_{\varepsilon}$ approaches 0.5 following a power-law decay $C_{\varepsilon}=24 \mathrm{Re}_{\lambda}^{-0.8}$, similar to Sreenivasan's ${ }^{33}$ prediction of decay of the form $C_{\varepsilon}=18.8 \mathrm{Re}_{\lambda}^{-1}$.

Moreover, an exponential decay of the turbulent energy dissipation rate versus the streamwise dimensionless abscissa $\mathrm{Re}_{z}$ was observed. In fact, moving downstream from the VG, the coherent structures become less and less energetic. In the present flow, it is observed that the dissipation rate $\varepsilon$ drops to low values at a distance of about $6 h$ downstream from the VG. Thus, it is recommended to add a new VG array $6 h$ from the previous one in order to maintain good micromixing.

Finally, the knowledge of the macro-, meso-, and microscales in the flow allows some insight into the turbulence structure of the complex flow generated by the VG and can provide some sizing criteria for multifunctional heat exchangers/reactors.

\section{ACKNOWLEDGMENTS}

C. Habchi wishes to thank G. Antar of the American University of Beirut for fruitful and enlightening discussions.

${ }^{1}$ K. B. M. Q. Zaman and J. K. Foss, "The effect of vortex generators on a jet in a cross-flow," Phys. Fluids 9(1), 106-114 (1997).

${ }^{2}$ S. Dong and H. Meng, "Flow past a trapezoidal tab," J. Fluid Mech. 510, 219-242 (2004).

${ }^{3}$ O. Lögdberg, J. H. M. Fransson, and P. H. Alfredsson, "Streamwise evolution of longitudinal vortices in a turbulent boundary layer," J. Fluid Mech. 623, 27-58 (2009).

${ }^{4}$ C. Habchi and J.-L. Harion, "Residence time distribution and heat transfer in circular pipe fitted with longitudinal rectangular wings," Int. J. Heat Mass Transfer 74, 13-24 (2014).

${ }^{5}$ A. Ghanem, T. Lemenand, D. Della Valle, and H. Peerhossaini, "Static mixers: Mechanisms, applications, and characterization methods-A review," Chem. Eng. Res. Des. 92(2), 205-228 (2014). 
${ }^{6}$ Y. Tsuji, "High-Reynolds-number experiments: The challenge of understanding universality in turbulence," Fluid Dyn. Res. 41, 1-17 (2009).

${ }^{7}$ S. B. Pope, Turbulent Flow (Cambridge University Press, Cambridge, 2000).

${ }^{8}$ A. N. Kolmogorov, "Local structure of turbulence in an incompressible viscous fluid at very high Reynolds numbers," Dok1. Akad. Nauk SSSR 30, 301-305 (1941).

${ }^{9}$ P. E. Dimotakis, "The mixing transition in turbulent flows," J. Fluid Mech. 409, 69-98 (2000).

${ }^{10}$ H. Fellouah and A. Pollard, "The velocity spectra and turbulence length scale distributions," Phys. Fluids 21, 115101 (2009).

${ }^{11}$ T. Burghelea, E. Segre, and V. Steinberg, "Elastic turbulence in von Karman swirling flow between two disks," Phys. Fluids 19(5), 053104 (2007).

${ }^{12}$ G. Antar, "Kolmogorov-kraichnan scaling in the inverse energy cascade of two-dimensional plasma turbulence," Phys. Rev. Lett. 91(5), 055002 (2003).

${ }^{13}$ G. P. Bewley, K. Chang, and E. Bodenschatz, “On integral length scales in anisotropic turbulence,” Phys. Fluids 24(6), 061702 (2012).

${ }^{14}$ J. Nedic, O. Supponen, B. Ganapathisubramani, and J. C. Vassilicos, "Geometrical influence on vortex shedding in turbulent axisymmetric wakes," Phys. Fluids 27(3), 035103 (2015).

15 J. Baldyga and J. R. Bourne, Turbulent Mixing and Chemical Reactions (Wiley, Chichester, 1999).

${ }^{16}$ C. Habchi, T. Lemenand, D. D. Valle, M. Khaled, A. Elmarakbi, and H. Peerhossaini, "Mixing assessment by chemical probe," J. Ind. Eng. Chem. 20, 1411-1420 (2014).

${ }^{17}$ F. Azizi and A. M. Al Taweel, "Turbulently flowing liquid-liquid dispersions. Part I. Drop breakage and coalescence," Chem. Eng. J. 166, 715-725 (2011).

${ }^{18}$ P. C. Valente and J. C. Vassilicos, “Universal dissipation scaling for nonequilibrium turbulence,” Phys. Rev. Lett. 108(21), 214503 (2012).

${ }^{19}$ R. K. Thakur, C. Vial, K. D. P. Nigam, E. B. Nauman, and G. Djelveh, "Static mixers in the process industries-A review," Chem. Eng. Res. Des. 81(7), 787-826 (2003).

${ }^{20}$ A. Ghanem, C. Habchi, T. Lemenand, D. Della Valle, and H. Peerhossaini, "Energy efficiency in process industry-Highefficiency vortex (HEV) multifunctional heat exchanger," Renewable Energy 56, 96-104 (2013).

${ }^{21}$ H. Mohand Kaci, C. Habchi, T. Lemenand, D. Della Valle, and H. Peerhossaini, "Flow structure and heat transfer induced by embedded vorticity,” Int. J. Heat Mass Transfer 53, 3575-3584 (2010).

${ }^{22}$ C. Habchi, T. Lemenand, D. D. Valle, and H. Peerhossaini, “Turbulence behavior of artificially generated vorticity,” J. Turbul. 11, N36 (2010).

${ }^{23}$ L. H. Benedict and R. D. Gould, “Towards better uncertainty estimates for turbulence statistics,” Exp. Fluids 22(2), 129-136 (1996).

${ }^{24}$ A. Høst-Madsen and C. Caspersen, "Spectral estimation for random sampling using interpolation," Signal Process. 46(3), 297-313 (1995).

${ }^{25}$ Y. H. Pao, "Structure of turbulent velocity and scalar fields at large wave-numbers," Phys. Fluids 8, 1063-1075 (1965).

${ }^{26}$ G. K. Batchelor, The Theory of Homogeneous Turbulence (Cambridge University Press, Cambridge, 1953).

27 J. L. Lumley, "Interpretation of time spectra measured in high-intensity shear flows," Phys. Fluids 8, 1056 (1965).

${ }^{28}$ K. R. Sreenivasan, "On the universality of the Kolmogorov constant," Phys. Fluids 7(11), 2778-2784 (1995).

${ }^{29}$ P. Jonas, O. Mazur, and V. Uruba, "On the receptivity of the by-pass transition to the length scale of the outer stream turbulence,” Eur. J. Mech., B: Fluids 19(5), 707-722 (2000).

${ }^{30}$ F. Peyrin and A. Kondjoyan, "Effect of turbulent integral length scale on heat transfer around a circular cylinder placed cross to an air flow," Exp. Therm. Fluid Sci. 26(5), 455-460 (2002).

${ }^{31}$ C. Habchi, T. Lemenand, D. D. Valle, and H. Peerhossaini, "Alternating mixing tabs in multifunctional heat exchangerreactor," Chem. Eng. Process.: Process Intensif. 49(7), 653-661 (2010).

${ }^{32}$ U. Frisch, Turbulence: The Legacy of A. N. Kolmogorov (Cambridge University Press, Cambridge, 1995).

${ }^{33}$ K. R. Sreenivasan, "On the scaling of the turbulence energy dissipation rate,” Phys. Fluids (1958-1988) 27(5), 1048-1051 (1984).

${ }^{34}$ P. Burattini, P. Lavoie, and R. A. Antonia, “On the normalized turbulent energy dissipation rate,” Phys. Fluids 17(9), 098103 (2005).

${ }^{35}$ H. Wang and W. K. George, "The integral scale in homogeneous isotropic turbulence," J. Fluid Mech. 459, 429 (2002).

${ }^{36}$ J. Jimenez, A. A. Wray, P. G. Saffman, and R. S. Rogallo, "The structure of intense vorticity in isotropic turbulence," J. Fluid Mech. 255, 65-90 (1993).

${ }^{37}$ Y. Kaneda, T. Ishihara, M. Yokokawa, K. Itakura, and A. Uno, "Energy dissipation rate and energy spectrum in high resolution direct numerical simulations of turbulence in a periodic box," Phys. Fluids 15(2), L21 (2003).

${ }^{38}$ T. Gotoh, D. Fukayama, and T. Nakano, "Velocity field statistics in homogeneous steady turbulence obtained using a high-resolution direct numerical simulation," Phys. Fluids 14(3), 1065 (2002).

${ }^{39}$ F. Azizi and A. M. Al Taweel, "Hydrodynamics of liquid flow through screens and screen-type static mixers," Chem. Eng. Commun. 198(5), 726-742 (2011). 